\title{
Morphological and functional disturbances of platelets induced by cryopreservation
}

\author{
H BAYTHOON, EGD TUDDENHAM, RA HUTTON \\ From the Katharine Dormandy Haemophilia Centre \& Haemostasis Unit, Department of Haematology, \\ Royal Free Hospital, London NW3 2QG
}

SUMMARY In vitro morphological and functional studies were carried out on platelets which had been cryopreserved in the presence of $5 \%$ dimethyl sulphoxide (DMSO). Overall loss of platelets was around $50 \%$. Those which survived freezing and reconstitution showed marked morphological deterioration, increase of procoagulant activity (PF3a) and a decrease in their aggregability and adenine nucleotide content. We conclude that if transfused, cryopreserved platelets are likely to be less effective than fresh platelets and may activate coagulation in vivo and that they should only be used when suitable fresh platelets are not available.

Thrombocytopenia resulting from bone marrow depression is a frequent complication of the newer chemotherapeutic regimens used in the management of haematological malignancies. To minimise the risks of haemorrhagic complications in such cases, supportive therapy in the form of prophylactic platelet infusions is advisable. The increased demand for platelet concentrates has highlighted a number of complications with this type of therapy. Firstly, the rapid deterioration of viability of stored platelets leads to considerable wastage and sometimes to difficulty in meeting demands at weekends and during holiday periods. Secondly, since most platelet infusions are given without cross-matching for HLA-groups at least, the continued effectiveness of such therapy may be compromised by the development of antiplatelet antibodies by the recipient.

To overcome these problems the preparation and use of frozen platelets has been suggested ${ }^{1}$ since this would prolong the storage time sufficiently to enable a bank of HLA-typed platelets to be established and may, in some instances, enable a store of autologous platelets to be laid down during a patient's remission, ready for use should they be required during any subsequent relapse. ${ }^{2}$ Various freezing procedures have been used and the subject has been recently reviewed. ${ }^{3}$ While there is no generally approved methodology the use of dimethyl sulphoxide (DMSO) as a cryoprotectant appears to be more widely used than glycerol or other substances.

Platelet freezing programmes are currently being

Accepted for publication 18 January 1982 pursued at a number of centres throughout the world, but in many cases the efficacy of the product is determined either empirically or by using tests, the predictive value of which is difficult to establish. Moreover, few if any attempts are made to protect the recipients against the prothrombotic consequences of infusing large numbers of damaged or even disrupted cells. ${ }^{4}$

In this study we have assessed the in vitro platelet function of frozen platelet concentrates in a number of ways in order both to determine the extent of the damage caused by freezing and as a form of quality control for the procedures involved in platelet cryopreservation.

\section{Material and methods}

\section{PREPARATION OF FROZEN PLATELETS}

The method used was basically that described previously $^{2}$ with minor modifications recommended by other workers to improve the viability of the product. Venous blood was anticoagulated with one tenth volume of citrate-phosphate dextrose (CPD), prepared according to Dacie and Lewis ${ }^{5}$ in plastic bottles or bags. The blood was centrifuged immediately at $22^{\circ} \mathrm{C}$.for $15 \mathrm{~min}$ at $150 \mathrm{~g}$. The platelet-rich plasma (PRP) was removed and one tenth volume of acid citrate dextrose (ACD) was added to lower the $\mathrm{pH}$ to around 6.8. The PRP and the residual blood were recentrifuged at $22^{\circ} \mathrm{C}$ for $15 \mathrm{~min}$ at $1500 \mathrm{~g}$. Approximately $90 \%$ of the platelet-poor plasma (ACD-PPP) was removed and the platelet button was gently resuspended in the remainder. The platelet-poor plasma from the CPD blood (CPD- 
PPP) was removed and stored at $-40^{\circ} \mathrm{C}$ until required. An equal volume of ACD-PPP containing $10 \%$ (vol/vol) of DMSO was added slowly (10-15 $\min$ ) and with continuous gentle agitation to the platelet concentrate giving a final DMSO concentration of $5 \%$. The platelet count in these mixtures was approximately $1.5-2.0 \times 10^{\%} / \mathrm{ml}$. The platelet concentrates were then frozen at $1^{\circ} \mathrm{C} / \mathrm{min}$ to $-80^{\circ} \mathrm{C}$ in a programmable temperature controller (type PTC 200, planer Product Ltd) and stored in liquid nitrogen at approx $-120^{\circ} \mathrm{C}$ until needed.

The platelet concentrates were placed in a $37^{\circ} \mathrm{C}$ water bath until they had just thawed (about $5 \mathrm{~min}$ ). For functional studies, the suspensions were washed to remove the DMSO, using a combined centrifugation-gel filtration method ${ }^{6}$ but without adenosine and were resuspended in an equal volume of CPD-PPP.

At each stage in the above process an aliquot of the platelet suspension was set aside for platelet count and for functional and morphological studies.
PLATELET FUNCTION TESTS

Platelet counts were performed by the method of Brecher and Cronkite ${ }^{7}$ and platelet aggregation by the turbidometric method. ${ }^{8}$ Platelet-rich plasma $(0.45 \mathrm{ml})$ was stirred at $1000 \mathrm{rpm}$ in an aggregometer at $37^{\circ} \mathrm{C}$ with $0.05 \mathrm{ml}$ of either adenosine diphosphate (ADP), adrenaline (Sigma) collagen suspension (Horm-Chemie) or Ristocetin (Lundbeck). After three minutes, the fall in the optical density (OD) of the PRP was measured and expressed as a percentage of the OD difference between PRP and PPP. ${ }^{9}$ For ADP, the extent of the primary wave was also determined by measuring the fall in OD of PRP one minute after addition of the agonist.

Platelet factor 3 availability (PF3a) was measured by a modification of the method of Spaet and Cintron $^{10}$ in which the Stypven time of PRP is measured either directly or after incubation of PRP for $20 \mathrm{~min}$ at $37^{\circ} \mathrm{C}$ with kaolin and quantified by comparison with the Stypven time of PRP in which the platelets have been completely disrupted by repeated freez-

Table 1 Platelet aggregability at stages during cryopreservation. All results expressed as mean $\pm \operatorname{SD}(n=7)$

\begin{tabular}{|c|c|c|c|c|c|}
\hline \multirow{3}{*}{$\begin{array}{l}\text { Aggregating agent } \\
\text { (final conc) }\end{array}$} & & \multicolumn{4}{|c|}{ Platelet aggregation (\%) } \\
\hline & & \multirow[t]{2}{*}{$C P D-P R P$} & \multirow[t]{2}{*}{$A C D-P R P$} & \multicolumn{2}{|l|}{ DMSO-PRP } \\
\hline & & & & without freezing & with freezing \\
\hline ADP & $3 \mu \mathrm{mol} / \mathrm{l}$ & $\begin{array}{c}65 \pm 14 \cdot 5 \\
(35 \pm 10 \cdot 0)\end{array}$ & $61 \pm 13 \cdot 0$ & $57 \pm 14 \cdot 0$ & $\begin{array}{l}20 \pm 4 \cdot 0 \\
(7 \pm 6 \cdot 0)\end{array}$ \\
\hline $\begin{array}{l}\text { Adrenaline } \\
\text { Collagen } \\
\text { Ristocetin }\end{array}$ & $\begin{array}{l}3 \mu \mathrm{mol} / \mathrm{l} \\
2 \mathrm{mg} / \mathrm{ml} \\
1.25 \mathrm{mg} / \mathrm{ml}\end{array}$ & $\begin{array}{r}61 \pm 16 \cdot 5 \\
76 \pm 12 \cdot 5 \\
179 \pm 10 \cdot 0\end{array}$ & $\begin{array}{l}55 \pm 11 \cdot 5 \\
73 \pm 8 \cdot 5 \\
77 \pm 7 \cdot 5\end{array}$ & $\begin{array}{l}38 \pm 18 \cdot 5 \\
73 \pm 14 \cdot 0 \\
77 \pm 10 \cdot 5\end{array}$ & $\begin{aligned} 6 & \pm 7 \cdot 0 \\
32 & \pm 8 \cdot 5 \\
37 & \pm 9 \cdot 5\end{aligned}$ \\
\hline
\end{tabular}

Results in parentheses are the \% aggregation one minute after addition of ADP.

Table 2 Platelet factor 3 concentrations at stages during cryopreservation. All results expressed as mean \pm SD $(\mathrm{n}=7)$

\begin{tabular}{lccll}
\hline & \multicolumn{2}{l}{ Platelet factor 3 availability (\%) } & & \\
\cline { 2 - 4 } & CPD-PRP & ACD-PRP & DMSO-PRP & with freezing \\
\cline { 3 - 5 } & & & without freezing & $32 \cdot 2 \pm 18 \cdot 1$ \\
Basal & $1 \cdot 01 \pm 0 \cdot 35$ & $1 \cdot 21 \pm 0.29$ & $1 \cdot 24 \pm 0 \cdot 22$ & $33.8 \pm 16 \cdot 2$ \\
\hline
\end{tabular}

Table 3 Platelet adenine nucleotide concentrations at stages during cryopreservation. All results expressed as mean \pm SD $(\mathbf{n}=7)$

\begin{tabular}{|c|c|c|c|c|}
\hline & \multicolumn{4}{|c|}{ Nucleotides (nmol/10 $0^{8}$ platelets) } \\
\hline & \multirow[t]{2}{*}{$C P D-P R P$} & \multirow[t]{2}{*}{$A C D-P R P$} & \multicolumn{2}{|l|}{$D M S O-P R P$} \\
\hline & & & without freezing & with freezing \\
\hline $\begin{array}{l}\text { Total } \\
\text { ATP } \\
\text { ADP } \\
\text { ATP/ADP ratiơ }\end{array}$ & $\begin{array}{r}10.70 \pm 2.56 \\
6.33 \pm 1.10 \\
4.37 \pm 1.51 \\
1.45 \pm 0.11\end{array}$ & $\begin{array}{r}10.20 \pm 2.17 \\
6.50 \pm 2.15 \\
3.70 \pm 0.55 \\
1.76 \pm 0.66\end{array}$ & $\begin{array}{r}10.60 \pm 1.61 \\
6.23 \pm 1.90 \\
4.37 \pm 0.67 \\
1.43 \pm 0.70\end{array}$ & $\begin{array}{l}7.50 \pm 2.20 \\
4.50 \pm 1.20 \\
3.00 \pm 1.29 \\
1.50 \pm 0.41\end{array}$ \\
\hline
\end{tabular}




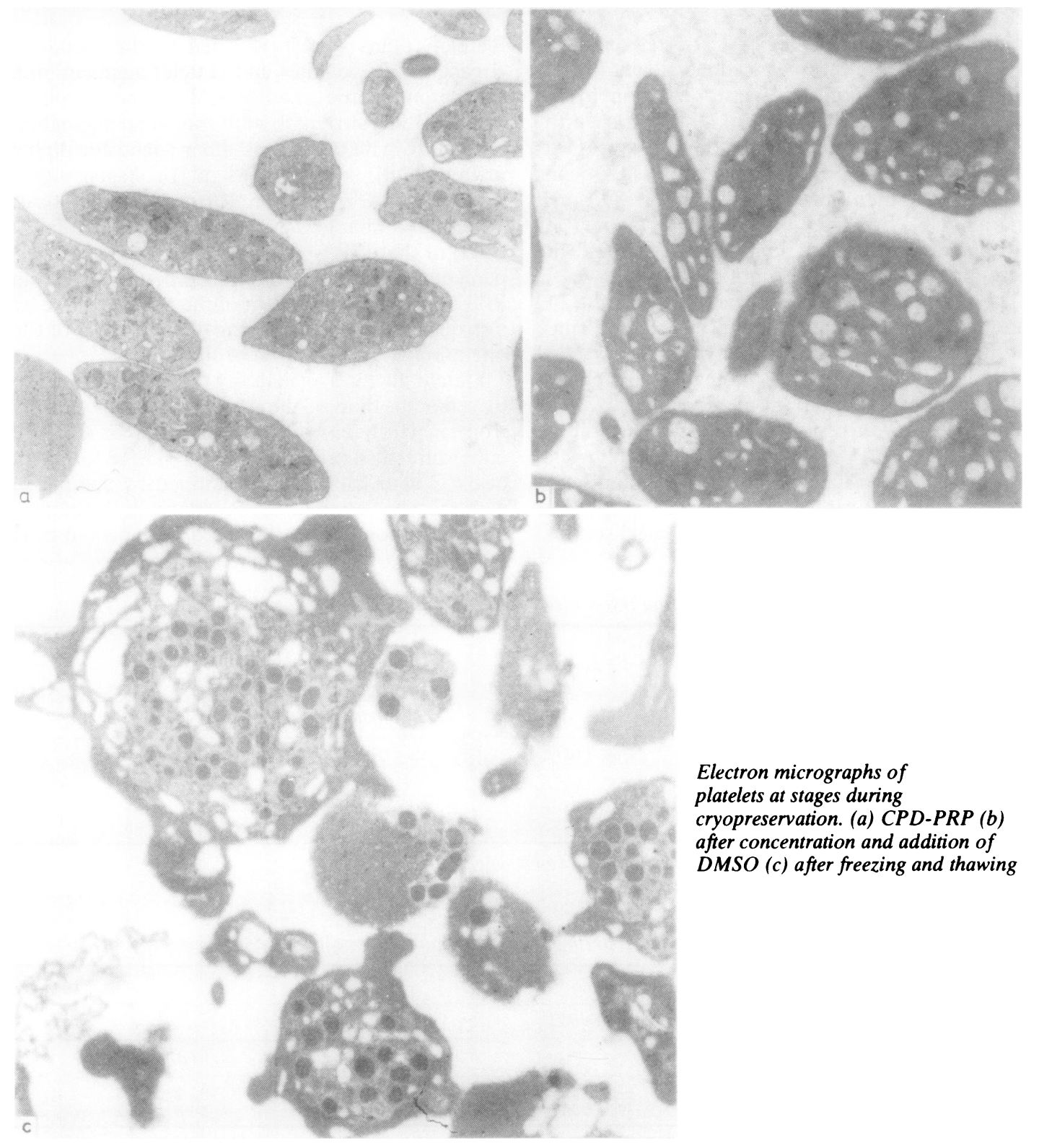

ing and thawing.

Platelet adenine nucleotide concentrations were measured by the bioluminescence method as described previously. " Results were statistically analysed using the paired $t$ test.

\section{ELECTRON MICROSCOPY}

One volume of platelet suspension was fixed for two hours at room temperature with four volumes of $1 \%$ glutaraldehyde in cacodylate-buffered saline. The platelets were sedimented by centrifugation, washed twice in the buffer, post-fixed in $1 \%$ osmium tetroxide for one hour and processed as previously described. ${ }^{6}$

\section{Results}

Recovery of platelets was monitored on five occasions. There was a small loss (mean 7\%, range 3$14 \%$ ) during the concentration stage but most of the 
loss (mean $33 \%$, range $24-45 \%$ ) occurred during the freezing/thawing stage with a further moderate fall (mean $7.5 \%$, range $2-12 \%$ ) during subsequent washing. Overall, about half the platelets survived the cryopreservation process.

\section{PLATELET AGGREGATION STUDIES}

There was no significant difference in platelet aggregability between blood anticoagulated with CPD or with $3.8 \%$ trisodium citrate. Because low pH ACD or DMSO completely abolish platelet aggregation, these substances had to be removed by washing before functional studies could be carried out. Table 1 compares the aggregation responses after washing, of ACD-PRP or DMSO-treated PRP, with and without freezing/thawing, with those of fresh CPD-PRP. Only the freezing and thawing process had any permanent inhibitory effect on aggregability. Responses using ADP or adrenaline as agonist were particularly affected, and with ADP especially, the initial rate of aggregation was decreased.

PLATELET FACTOR 3 AVAILABILITY (TABLE 2) Basal levels (no kaolin) of PF3a in washed ACDPRP and DMSO-PRP were only marginally higher than those in the original CPD-PRP and the additional amounts generated on incubation with kaolin were also similar. In contrast, markedly increased basal PF3a levels were found in washed, cryopreserved platelets and little or no further rise could be induced by kaolin activation.

\section{PLATELET ADENINE NUCLEOTIDES (TABLE 3)}

Nucleotide concentrations were similar at all stages prior to freezing. However, cryopreservation resulted in a statistically significant decrease $(p<$ 0.02) in ATP and ADP concentrations although levels were still within the normal range.

\section{MORPHOLOGICAL STUDIES}

Compared to freshly fixed platelets (Fig. a), those which had been processed to the DMSO stage before fixation often showed some loss of discoid shape with moderate vacuolation (Fig. b), the latter probably being due to dilatation of the open canalicular system. Even when the normal discoid shape was maintained during this stage it was lost on subsequent cryopreservation when more marked morphological changes were invariably observed (Fig. c). Plasma membrane damage after freezing was particularly obvious, many cells having a fuzzy outline with numerous large pseudopods devoid of granules. The cell granule complement, including dense bodies, appeared to be relatively normal however.

\section{Discussion}

The present study shows that platelets cryopreserved in 5\% DMSO using existing methodology, exhibited marked morphological and functional abnormalities. Alterations in the plasma membrane of the platelets were clearly visible in electron micrographs and were associated with the development of a potent, phospholipid-related procoagulant activity, referred to as PF3.

The ability of cryopreserved platelets to undergo the normal aggregation responses to physiological agonists such as ADP and collagen, upon which in vivo platelet plug formation depends, was also impaired. This was probably related to the plasma membrane changes since primary aggregation which depends on surface-related events, was particularly inhibited whereas the granule content and the releasable pool of adenine nucleotides through which secondary aggregation is mediated, were relatively unaffected.

Practically all of these disturbances were due to the freezing-thawing step since only minimal changes were induced by DMSO or by the washing procedure used to remove the cryoprotectant.

Morphological abnormalities similar to those described above have been published previously ${ }^{12} 13$ but because of the technical difficulties of applying to cryopreserved platelets the tests routinely used to evaluate platelet function, these have been rejected by other workers in favour of tests which, though simpler to perform, may be of more questionable physiological significance. For example, serotonin (5HT) uptake by platelets has been advocated by some, ${ }^{14}{ }^{15}$ despite the fact that since this property is retained by isolated platelet dense bodies ${ }^{16}$ it cannot be regarded as a measure of the cell's intactness. Similarly, the hypotonic shock response of cryopreserved platelets may not always correlate with platelet survival. ${ }^{17}$

Whatever the methods used to assess in vitro the viability of cryopreserved platelets the almost universal conclusion has been that they respond markedly less well than fresh platelets. Clearly, however, the critical test is their ability to circulate, survive and function normally when reinfused. Several studies have shown a modest clinical improvement associated with an increment in the platelet count in recipients of cryopreserved platelets. However, the platelet recovery rates have been extremely variable and rarely more than half those achieved with fresh platelets, even though the platelets which do remain in the circulation may have a close to normal survival. In vivo functional studies have often been uncontrolled or evaluated subjectively, or in some instances, omitted altogether. Partial correction of 
the bleeding time has been noted ${ }^{2}$ but where it has been possible to recalculate the data applying the formula developed by Harker and Slichter ${ }^{18}$ which relates bleeding time to platelet count, the shortening was almost invariably less than expected.

In addition to these iatrogenic functional abnormalities, some types of leukaemia are associated with intrinsic platelet defects which may persist during remission and these will further limit the value of frozen autologous platelet infusions in such cases.

Finally, scant attention has been paid to the possibility of inducing a hypercoagulable state by transfusing large numbers of damaged cells. Although no cases of clinically overt thrombotic sequelae has been reported, Odink and Brand ${ }^{14}$ noted an increase in fibrinopeptide $A$ level in one of seven patients studied following infusion of cryopreserved platelets derived from approximately one unit of blood.

Thus we believe that the low functional activity of cryopreserved platelets coupled with the expensive and time-consuming manoeuvres involved in their production argue against their use for purposes of simple expediency and that they should only be used when transfusion of compatible platelets might be life-saving and suitable fresh platelets are not available.

We wish to thank Mr Alan Parsonson for his help with the freezing process and Miss Jacqueline Lewin for preparing the electron micrographs. We are grateful to the staff of the Royal Free Hospital who generously donated blood for these studies, and to Mrs Leela Gandhi for secretarial assistance.

\section{References}

${ }^{1}$ Cohen P, Gardner FH. Platelet preservation. IV. Preservation of human platelet concentrates by controlled freezing in a glycerol medium. $N$ Engl $J$ Med 1966;274:1400-7.

${ }^{2}$ Schiffer CA, Aisner J, Wiernik PH. Frozen autologous platelet transfusion for patients with leukaemia. $N$ Engl J Med 1978;299:7-12.

${ }^{3}$ Meryman HT, Burton JL. Cryopreservation of platelets. In: Greenwalt TJ, Jamieson GA, eds. The blood platelet in transfusion therapy. New York: Alan R Liss, 1978.

${ }^{4}$ Donati MB. The laboratory diagnosis of acquired defects of haemostasis. In: Thomson J, ed. Blood coagulation and haemostasis: a practical guide. Edinburgh: Churchill-Livingstone, 1980.

${ }^{5}$ Davie JV, Lewis SM. Practical haematology 5th ed. Edinburgh: Churchill-Livingstone, 1975.

' Hutton RA, Howard MA, Deykin D, Hardisty RM. Methods for the separation of platelets from plasma. A comparison of functional and morphological integrity. Thrombosis et Diathesis Haemorrhagica 1974;31:119-32.

${ }^{7}$ Brecher G, Cronkite EP. Morphology and enumeration of human blood platelets. J Appl Physiol 1950;3:365-77.

${ }^{8}$ Born GVR. Aggregation of blood platelets by adenosine diphosphate and its reversal. Nature 1962;194:927-9.

${ }^{9}$ Hutton RA, Mikhailidis DP, Dormandy KM, Ginsburg J. Platelet aggregation studies during transient hypoglycaemia. J Clin Pathol 1979;32:434-8.

${ }^{10}$ Spaet TH, Cintron J. Studies on platelet factor 3 availability. Br J Haematol 1965;11:269-75.

" Hardisty RM, Hutton RA, Montgomery D, Rickard S, Trebilcock $H$. Secondary platelet aggregation: a quantitative study. Br J Haematol 1970;19:307-19.

${ }^{12}$ Crowley JP, Rene A, Valeri CR. Changes in platelet shape and structure after freeze preservation. Blood 1974;44:599-603.

${ }^{13}$ Khan RA. Biochemical changes in frozen platelets. In: Greenwalt TJ, Jamieson GA, eds. The blood platelet in transfusion therapy. New York: Alan R Liss, 1978:167-80.

14 Odink J, Brand A. Platelet preservation. V. Survival, serotonin uptake velocity and response to hypotonic stress of fresh and cryopreserved human platelets. Transfusion 1977:17:203-9.

15 Tandy NP, Taylor MA. Platelet concentrates for transfusion: control of production and storage. Med Lab Sci 1980;37:127-36.

${ }^{16}$ Rendu F, Lebret M, Caen JP. Isolation of dense bodies from human blood platelets on metrizamide gradient. Thromb Haemost 1981;46:133.

17 Valeri CR, Feingold H, Marchionni LD. The relation between response to hypotonic stress and the ${ }^{51} \mathrm{Cr}$ recovery in vivo of preserved platelets. Transfusion 1974;14:331-7.

${ }^{18}$ Harker LA, Slichter SJ. The bleeding time as a screening test for evaluation of platelet function. $N$ Engl $J$ Med 1972;287:155-9.

Requests for reprints to: Dr RA Hutton, Katherine Dormandy Haemophilia Centre and Haemostasis Unit, Dept of Haematology, Royal Free Hospital, London NW3 2QG, England. 